\title{
REPORT ON THE HEALTH OF THE ARMY 1965
}

\author{
A: H. GOULD, B.Sc., F.I.A. \\ Ministry of Defence (Army), AMD (Stats)
}

IN previous years it has been the practice for the statistical tables relating to the Health of the Army to be published officially by the Army Department. Covering these tables a short statistical summary was presented but it was not the practice for any detailed statistical review to be made. In respect of the figures for the year 1965 a break was made with past practice in that the statistical tables were not produced as an official publication, but were merely circulated as a report of AMD Stats branch.

There is no doubt that the figures presented in this report are likely to be of interest in general to the readers of the Journal, the more so when given a reasonably detailed statistical appraisal. The intention of this article is to provide just such an appraisal, although of course it is impossible to reproduce the figures themselves. Nevertheless since this report is, in spite of its limited publication this year, available to all those who may be interested in examining the results, the following discussion will largely assume that the figures concerned are known to the reader.

There is one feature of the medical experience presented in the tables of the report which must be emphasized from the outset; that is, the figures are derived entirely from the experience of hospitals and other medical units and hence relate to admissions to these units and not to all sickness occurring in the Army. Excluded therefore are those who are treated at unit medical officer level, together with those who are attended at home or on sick leave. The result is that in terms of rates of sickness and of non-effectiveness there is a possible under-statement, so that to be strictly accurate the tables should be headed "Rates of Sickness, etc. determined at medical units."

The first group of tables presented gives a summary of the admission rates by Commands (for both British Army males and females) and the comparison of these rates yearly over the past five-year period. As the experience of British Army males forms the greatest part of the medical sickness story, the comments below will relate to the male experience only, unless specific reference is made to females.

The difference between Commands preserves the pattern observed in the past, with BAOR showing the most favourable rate (179.7 per thousand strength) and FARELF the highest (337.4 per thousand). Referring to the annual comparisons, the rates for the individual Commands all show a continuous improvement over this period, although not all are lower than the corresponding 1964 figures. It is perhaps noteworthy that the one theatre not showing the same general improvement is the UK, for which the rates have been virtually consistent for the past four years. The next batch of tables presents the incidence of medical non-effectives, also by Commands, and an annual comparison over the five-year period. The same " between Command" differences as were observed in considering admission rates are seen here; in fact the differences are accentuated. Thus the average medical non-effective rate for the UK is 12.4 per thousand, whilst for BAOR the corresponding figure is 5.5 This reflects the fact that the duration of stay in hospitals in the UK is considerably longer than that in BAOR. Working from the admission rates and the non-effective rates, a figure of 16.5 days' stay is obtained for the UK compared with 11.5 for BAOR. Even the figure for FARELF, in terms of 
average duration, is less than that for UK, since with an average medical non-effective rate of 10.5 per thousand the corresponding duration of stay is approximately 11 days. Whilst a large part of this comparatively long duration of stay in the UK can be explained by return of reference cases, which are generally of long duration, to hospitals in this country and there is also the existence of the Army Medical Rehabilitation Unit with its long duration cases, the difference warranted the inception of : an investigation by AMD Stats, the results of which tend to confirm that even in general hospitals the average stay is longer in UK than overseas.

Another feature in the comparison of the medical non-effective rates for the past five years is that for the first time there has been a significant decrease in these. This is more particularly noticeable for females than for males. As this decrease is far greater than that which could be accounted for by the slight reduction in admission rates, the only cause must be a change in the duration of stay pattern which again has no obvious explanation.

After a short table quoting average bed occupancy within the different theatres, the next main group of tables gives the breakdown by disease of the total admissions in each theatre. Whilst comparable figures for previous years are not presented in the Report, it can be stated that the same general pattern by disease both within each Command and between Commands is preserved. Thus tonsillitis and pharyngitis is a very large contributor particularly in the UK, with skin conditions also forming a large part of the sickness experience. As expected the latter is of particular weight in FARELF and Near East with rates of 20.3 per thousand and 23.6 per thousand respectively, and even in BAOR 9.1 per thousand. "In the Caribbean Area, the rate was 59.0 per thousand, although the number at risk was comparatively small". One of the largest single contributors to the total for the year in the UK is common cold ranked with a rate of 10.2 per thousand, but in BAOR, with a similar temperate climate, which might be expected to show similar patterns of sickness, the rate was only 1.9 per thousand. Diarrhoea and enteritis figures very largely in the Middle East and Near East experience, with rates of 25.7 per thousand and 16.7 per thousand respectively, by far the largest single cause of sickness in the former. A feature which has persisted over the past few years, which could obviously be related to the prevailing local conditions, is the high admission rate for psychiatric conditions in both the Near, East and Middle East. Another feature which is worth comment is the figure for venereal disease in FARELF. With a rate of 23.2 per thousand it is still the largest item in the FARELF pattern of diseases yet is far less than previously recorded, the figure for 1964 being 43.1. The same table also presents the injury rates for the different theatres, though a comparison with the experience of previous years is not shown. Nevertheless, it is worth mentioning that by comparison with the injury figures for 1964, the total rate, for the first time for many years, has shown a slight decrease, not constant between all theatres, but of the order of 5 per cent overall. The decrease is most marked in BAOR where the rate has fallen to 51.4 per thousand.

The breakdown by diseases for British Army females is of necessity confined mainly to UK and BAOR, since the strength of the Women's Corps in other theatres is very small. For the two main theatres considered certain features comparable to the male experience could be seen. For example, tonsillitis and pharyngitis was the largest single cause of sickness. Gynaecological conditions naturallv form a large part of the experience, 
but in the UK there is also a large admission rate for appendicitis- -17.1 per thousand. Skin conditions, common cold and diarrhoea and enteritis maintain the same relative position as in the male experience, all at a slightly higher level than for the males. Again, as could be expected, the proportion of injuries to all diseases is far less for females than for males, accounting for only about seven per cent of the total admission rate compared with 22 per cent for the males.

In support of the theatre figures, area breakdowns are quoted for both FARELF and Near East. In the former, the difference in levels between Malaya, Singapore, Hong Kong and Borneo, in that order, is very apparent. This ,however, is a somewhat spurious comparison, since the figure for Borneo admissions does not include those more serious cases arising within that territory which were evacuated to Singapore for treatment. The figure of 165 per thousand for Borneo is therefore an understatement of the actual incidence of sickness in that theatre. It has been possible to prepare within AMD Stats a figure of the evacuations, and if this is added back to the Borneo figure mentioned above, a total of approximately. 280 per thousand is obtained. This is still satisfactorily low and represents a feature observed in most collections of medical statistics during operations; that is, that sickness rates as such tend to be low where operations are in progress. In the area table for FARELF the picture of the reduction of venereal disease admissions for the year is shown for all areas; against this striking decrease the table at the same time shows an increase in the total rate for venereal disease and hence an increase in cases not admitted to hospital. How much is due to change in experience or how much is due to a change in the mode of treatment cannot be determined at this point.

The similar breakdown for areas in Near East shows differences between the areas, with Malta showing up particularly favourably, Cyrenaica and Tripolitania particularly unfavourably, although both of these do show some improvement over the past years. One disquieting feature of both Tripolitania and Cyrenaica experience is the large injury rate (72 per thousand Tripolitania, 90 per thousand Cyrenaica). It is not possible to relate these figures to basic causes, although it is unlikely that the explanation lies in the unfavourable rate of road traffic accidents. However, the Tripolitanian rate shows a considerable improvement over the corresponding figure for 1964 (109 per thousand).

Tables of medical evacuation follow, showing in the first place, breakdown by Command and disease and then comparison of figures over the past 5 years. From all theatres the rate of evacuation was lower in 1965 than in 1964. From BAOR the main causes for evacuation were psychiatric conditions and injuries with rates of 2.6 per thousand and 2.8 per thousand respectively. From FARELF injuries formed by far the largest single cause ( 3.9 per thousand), with skin conditions of next importance (1.2 per thousand). In this theatre, evacuation resulting from psychiatric conditions amounted to only 0.7 per thousand strength. The Middle East and Near East tended to repeat the BAOR experience, with psychiatric conditions and injuries being the two major causes of evacuation in both theatres. Summaries of invalidings follow. Possibly the most interesting is the last of these tables which compares the annual rates for the past 20 years. Its main feature is to demonstrate that the invaliding rate for all causes has been virtually unchanged, with minor variations, since 1951 (an average of about 16 per thousand). The same constancy is observed in the rate of invaliding through psychiatric conditions, the main cause of invaliding, the average rate of this disorder being 4.4 per thousand for the same period, 1951-1964. In 1965 the actual rates were 16.2 per thousand for 
total invaliding and 4.2 per thousand for psychiatric disorders. Apart from psychiatric disorders the only causes of major importance were gastric and duodenal ulcers, 1.5 per thousand strength; disease of the ear, 1.1 per thousand; diseases of the bones and joints, 0.8 per thousand; deformities of the foot, 0.7 per thousand; and diseases of the skin 0.7 per thousand. Mention should be made here of the experience regarding invaliding of British Army females, in particular the strikingly high invaliding rate due to psychiatric disorders. At 23.1 per thousand strength this accounted for 79.4 per cent of all invalidings, the total invaliding rate being 29.1 per thousand.

The table of deaths by theatres and diagnosis which follows emphasises the high relative mortality rate through injury. Of a total of 328 deaths it is observed that 233 were the result of injury. This rate has been preserved fairly constantly over the years and there is no doubt that it includes a large number of road traffic accident deaths. Deaths arising as a result of heart diseases showed another annual increase; from a low figure of 22 in 1963 they have risen to 40 in 1965. Deaths through neoplasms remain at about the same level, the figure of 31 being shown for 1965 compared with an average of 32 over the past five years. The only other single cause of any real significance is vascular lesions affecting the central nervous system which were responsible in 1965 for six deaths.

The summary table of dental treatment follows. These figures are of interest, for whilst the treatment rates do not vary greatly between the different theatres, (from 1483 per thousand in FARELF to 1164.5 per thousand in Near East), the nature of the treatment varied considerably. Thus, in FARELF, the number of teeth extracted was 425.8 per thousand, and in the UK 418.0 per thousand, whilst in the Middle East the figure was 229.8 per thousand. The variation in the number of teeth conserved is even greater, a figure of 1191.3 per thousand being observed in the UK but only 491.9 per thousand in BAOR. Similar wide variation is shown in virtually all treatments listed and an investigation into the causes of these differences might prove an interesting field for research.

The table of heights and weights of recruits in 1964 follows, giving the averages by age. It is regretted that the figures in this table are in error and a corrigendum is being prepared for inclusion in the Report on the Health of the Army 1966. This table shows the expected variation of average weight with age and the normal variability about the average weight with the exception of a very high average weight for recruits aged 30 years and over. This has been so apparent that a special investigation was carried out by AMD Stats to determine the cause for this increase. It appears that in the year 1964, some special enlistments of overweight recruits were accepted, and particularly at ages over 30 years there was a sufficient number to bias the average weight for the whole of this group.

The subsequent tables give a breakdown of admissions by detailed diagnosis and age groups. They give the annual rates per thousand strength and the average duration of stay in days separately, for all major theatres. Since they are derived from the F's Med 14 received, there is an inevitable delay in compiling them, so that the published tables are, in fact, related to the experience of the year 1962. They form too large a volume of information for description here, but are of most value to readers concerned with detailed investigations into incidence of diseases, length of sickness, variation of sickness, experience with age and similar projects. The tables quoting the average duration of stay are particularly interesting in indicating the relative severity of various diseases 
in terms of time of medical attention in units. A comparison between injury treatment time and disease treatment time shows that without exception the duration of stay for injuries is greater than that for diseases, in spite of the fact that the former includes a large number of relatively minor incidents such as lacerations, superficial injuries and sprains and strains.

Finally, a table which appears for the first time is presented-a breakdown of injury admissions by nature and external cause. This illustrates the pattern which has been observed fairly constantly over the past years; one-fifth of accidents occurred in land transport, one-fifth during athletics and sport, and two-fifths through falls and miscellaneous causes. The remaining one-fifth was made up of small numbers of accidents arising from other specified causes. The relation of the cause to the nature of the injury is interesting. Thus in land transport accidents the high proportion of head injury, with or without fracture of the skull, and fracture of the limbs amount to 3.0 and 2.2 per thousand strength respectively. In the athletics and sports group although the number, as expected, is large, the majority of these fall into the sprains group ( 3.2 per thousand) with 2.0 per thousand resulting in a fracture of limb.

This brief summary as a statement of the in-patient experience of sickness and injury in the Army is necessarily very sketchy. In many instances the figures quoted for the year, whilst of some interest, show their true importance only when compared with the figures of other years. For those who are interested in carrying out research or investigation involving these rates, reference to the complete tables and to the tables for preceding years is essential, and the above review can only begin to give a very brief picture of what is generally termed "The Health of the Army".

REPRESENTATIVE COLONEL COMMANDANT, R.A.M.C.

Major General W. A. Robinson, C.B., O.B.E., M.D., has been appointed Representative Colonel Commandant, R.A.M.C. for 1968.

\section{INDEX}

The Joint Index for 1967 of the Journal of the Royal Army Medical Corps and the Army Medical Services Magazine will be distributed with the April, 1968 issues.

\section{Preliminary Announcement-Corps Occasions for 1968}

Provisional dates are as follows:-

R.A.M.C. Officers' 'At Home' (4-6 p.m.)

5th July

R.A.M.C. Officers' Dinner (7.30 for 8 p.m.)

5 th July

Corps Sports

6th July

R.A.M.C. Depot and Old Comrades Church Parade and 'At Home' 7th July

Corps Golfing Society Summer Meeting

Swimming Gala

8th July

10th October 
Lieutenant-Colonel A. SIMPSON-SMITH, M.A. M.Chir., F.R.C.S. (Eng), R.A.M.C.

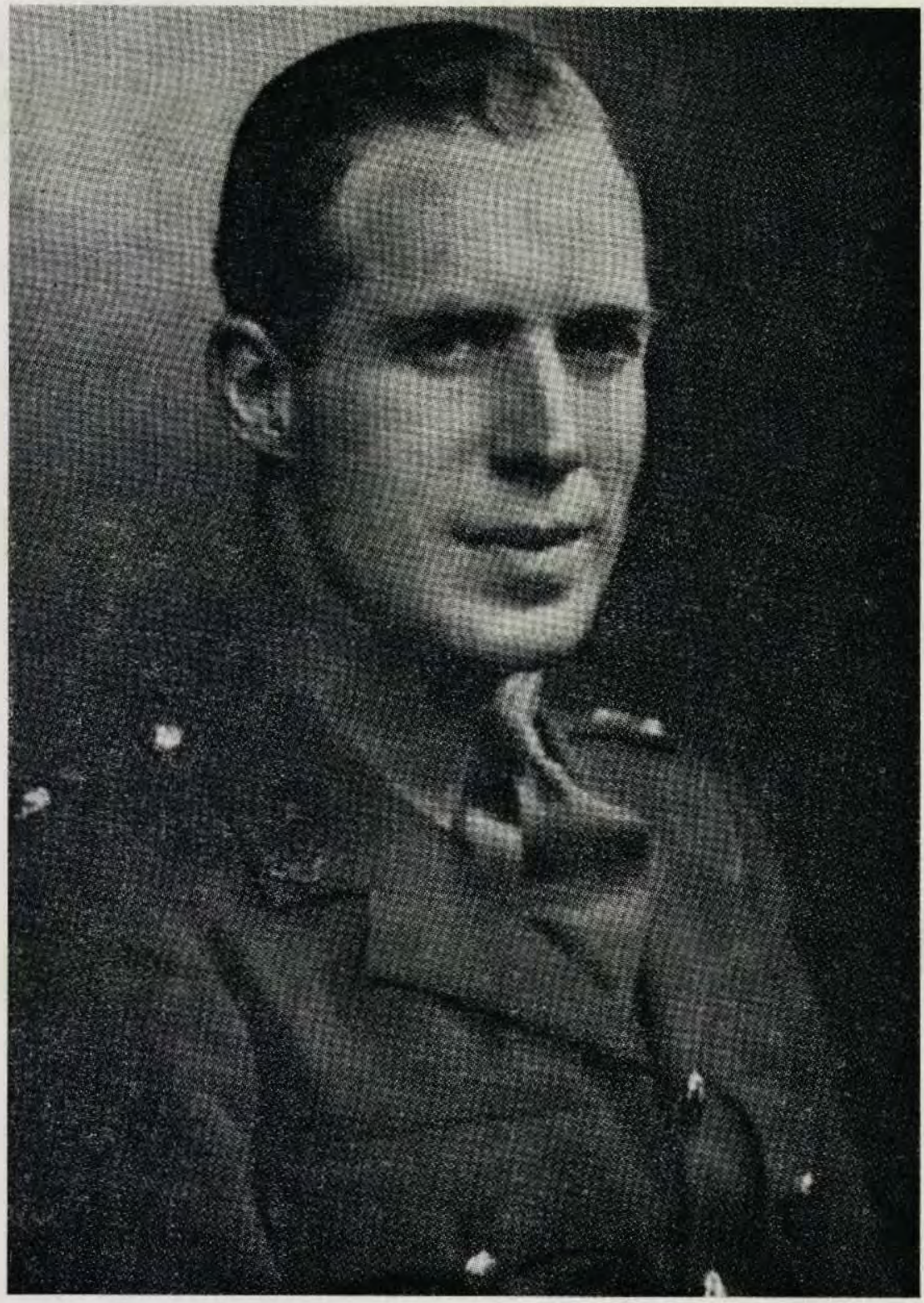

Fig. 1 